\title{
Análise crítica de ensaios clínicos aleatórios sobre fisioterapia aquática para pacientes neurológicos
}

\author{
Critical analysis of randomized clinical trials in aquatic physical \\ therapy applied to neurological patients
}

Rafaela Okano Gimenes ${ }^{1}$, Sissy Veloso Fontes ${ }^{2}$, Márcia Maiumi Fukujima ${ }^{3}$, Sandro Luis de Andrade Matas ${ }^{4}$, Gilmar Fernandes do Prado ${ }^{5}$

\section{RESUMO}

Objetivos: Revisão da literatura na busca de evidências da efetividade da fisioterapia aquática em pacientes com doença neurológica e análise crítica dos ensaios clínicos aleatórios (ECA). Método: Localização dos ensaios clínicos aleatórios através da busca em bases eletrônicas e análise crítica dos ECA utilizando a lista Delphi. Resultados: Dos 207 trabalhos localizados 157 foram recuperados. Destes, 22 foram utilizados, sendo apenas 6 ECA. Um trabalho apresentou o método de aleatorização, mostrando que este não é um método utilizado regularmente em estudos clínicos sobre este assunto, devendo estudos futuros utilizar-se deste método para a alocação dos pacientes. A maioria dos estudos apresentou grupos similares com relação aos indicadores prognósticos mais importantes dos pacientes e, todos os estudos apresentaram critérios de elegibilidade especificados, intenção de tratar e foram relatadas estimativas das medidas de tendência central e variabilidade nas medidas de desfecho primário propiciando, assim estudos de melhor qualidade. Conclusão: existem fracas evidências na literatura dos benefícios da fisioterapia aquática quando aplicada à pacientes com doenças neurológicas, sendo fundamental a elaboração de um ensaio clínico aleatório de grande amostra para avaliar o efeito da fisioterapia aquática em pacientes com doença neurológica específica.

Unitermos: Hidroterapia, Fisioterapia aquática, Doenças neurológicas.

Citação: Gimenes RO, Fontes SV, Fukujima MM, Matas SLA, Prado GF. Análise crítica de ensaios clínicos aleatórios sobre fisioterapia aquática para pacientes neurológicos. Rev Neurociencias 2005; 13(1):005-010.

\section{SUMMARY}

Purpose: to carry out a systematic literature review in order to find evidence of the efficiency of aquatic therapy for neurologically impaired patients with motor disorders; to critically analyze the randomized clinical trials (RCT) identified. Methods: Identification of RCT through electronic sources, and critical analysis of the RCT according to the Delphi checklist. Results: the method of randomization was found in only one out of six studies, which leads to the conclusion that this method is not used in a consistent manner by clinical studies on the topic. Thus, future research should employ the randomization method for the

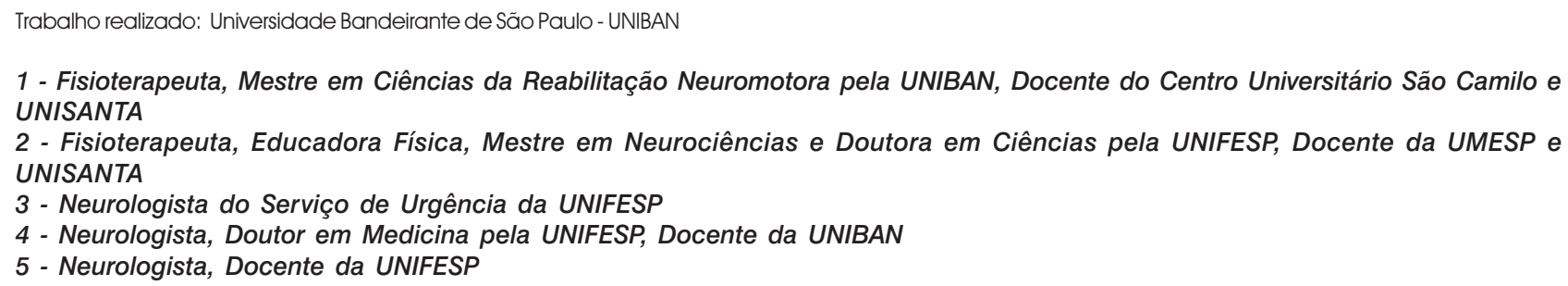


allocation of patients, for there are no restrictions for its usage in physiotherapeutic studies. In addition, the majority of the studies presented similar groups in terms of the most significant prognostic indicators of the patients, and all papers provided enough information about the eligibility criteria, intention to treat and statistical analysis (point estimates and measures of variability), which ensures the quality of the studies. Conclusion: there is no clear evidence in the literature about the benefits of aquatic physical therapy when applied to neurologically impaired patients with motor disorders. Given this gap in the literature, it becomes essential to develop a RCT in order to assess the effects of aquatic physical therapy on patients with specific neurological dysfunctions.

\section{Keywords: Hydrotherapy, Aquatic Physical therapy, Neurological Disease.}

Citation: Gimenes RO, Fontes SV, Fukujima MM, Matas SLA, Prado GF. Critical analysis of randomized clinical trials in aquatic physical therapy applied to neurological patients. Rev Neurociencias 2005; 13(1):005-010.

\section{INTRODUÇÃO}

Atualmente não há um consenso sobre a terminologia mais adequada para denominar o procedimento fisioterapêutico cinesioterapia, quando executado em meio aquático. Sendo assim, dos vários termos que vêm sendo utilizados (reabilitação aquática, hidrocinesioterapia, exercícios aquáticos), consideramos o termo "fisioterapia aquática" o mais indicado, por isso, o eleito neste trabalho para denominar o procedimento fisioterapêutico a ser estudado.

A fisioterapia aquática possui uma longa história e é tão importante atualmente quanto foi no passado. Hoje, com o crescimento de sua popularidade, os fisioterapeutas são encorajados a utilizarem a água aproveitando ao máximo suas propriedades.

Para que possamos compreender este recurso fisioterapêutico faz-se necessário o amplo conhecimento sobre as propriedades hidrostáticas, hidrodinâmicas e termodinâmica da água (mecânica de fluidos).

A imersão aquática possui efeitos fisiológicos relevantes que se estendem sobre todos os sistemas e a homeostase. Estes efeitos podem ser tanto imediatos quanto tardios, permitindo assim, que a água seja utilizada para fins terapêuticos em uma grande variedade de problemas orgânicos. A terapia aquática parece ser benéfica no tratamento de pacientes com distúrbios músculo-esqueléticos, neurológicos, cardiopulmonares, entre outros ${ }^{[1]}$.

Campion ${ }^{[2]}$ cita que apenas por meio da experiência clínica pode-se sugerir que a fisioterapia aquática seja um meio efetivo e prático para a recuperação de pessoas que estão com disfunções neurológicas, uma vez que poucas pesquisas têm sido realizadas.

Portanto, o foco deste estudo consiste em analisar apenas os trabalhos científicos baseados em en- saios clínicos aleatórios (ECA) de fisioterapia aquática em pacientes neurológicos com comprometimento motor, para averiguarmos se existem evidências científicas sobre os efeitos da fisioterapia aquática para estes pacientes.

Os ECA podem ser definidos como experimentos analíticos nos quais os investigadores alocam aleatoriamente os sujeitos elegíveis para o grupo de tratamento e grupo controle e, os resultados são avaliados comparando ambos os grupos.

Os objetivos deste trabalho abrangem: 1. Revisão da literatura na busca de evidências da efetividade da fisioterapia aquática em pacientes neurológicos com comprometimento motor; 2. Análise crítica dos ECA sobre fisioterapia aquática.

\section{MATERIAL E MÉTODO}

Foi realizada revisão abrangente da literatura, obedecendo aos seguintes critérios:

1 - Estratégias usadas para identificação dos estudos

Ensaios clínicos aleatórios relevantes foram identificados eletronicamente por busca nas bases de dados: Medline, Embase, Scielo, Lilacs, Cochrane Library, além da procura manual nas referências dos artigos. A pesquisa compreendeu o período de 1950 a 2004. A estratégia adotada e os descritores utilizados foram: randomized-controlled trial in pt, randomized-controlled-trials, random-allocation, double-blind-method, single-blind-method, clinicaltrial in pt, neurological disorders and hydrotherapy, neurological disorders and aquatic physical therapy; neurological disorders and exercise in water, neurological disorders and aquatic exercises, neurological disorders and water therapy, neurological disorders and water immersion, neurological diseases and hydrotherapy. 


\section{2 - Tipos de estudos}

Foram considerados todos ECA onde os grupos de estudo receberam tratamento com fisioterapia aquática, e os grupos controle receberam tratamento tanto de fisioterapia tradicional (cinesioterapia em solo) como a fisioterapia aquática ou natação adaptada.

\section{3 - Tipos de participantes}

Todos os pacientes dos estudos apresentavam doença neurológica com comprometimento motor (paralisia cerebral, poliomielite, lesão medular, traumatismo crânio-encefálico, acidente vascular cerebral, aneurisma cerebral e esclerose múltipla) de faixas etárias diversas (criança, adolescente, adulto e idoso).

\section{4 - Tipos de intervenção}

Foi considerado qualquer protocolo de intervenção fisioterapêutica aquática, que consistiu na aplicação de cinesioterapia em meio líquido, com movimentos passivos, ativos, ativo-resistidas, exercícios de coordenação motora, treino de marcha subaquática e nado livre, quando possível. Estes protocolos foram comparados com os seguintes grupos controle: que receberam intervenção de atividade física sem fins terapêuticos na água, cinesioterapia em solo e natação adaptada.

\section{5 - Aplicação dos critérios iniciais de inclusão}

Nesta primeira fase foi verificado se o estudo era ECA e analisada a ocultação da alocação, seguindo a classificação:

A. Medidas adequadas para o sigilo da alocação como aleatorização central, números seriados, envelopes, ou outras descrições de ocultação considerada adequada;

B. Estudos clínicos com descrição vaga da ocultação da alocação e que não se enquadram na categoria anterior;

C. Ensaios clínicos controlados, em que o método de alocação não era oculto.

6 - Qualidade metodológica dos estudos incluídos

De acordo com Verhagen et al ${ }^{[3]}$ atualmente, as revisões sistemáticas contam com uma avaliação mais fidedigna da qualidade metodológica dos ECA.

A lista de Delphi foi desenvolvida com a finalidade de avaliar a qualidade dos ECA, a fim de condu- zir uma revisão sistemática adequada. É composta por oito itens, e as respostas são apresentadas nas formas de "sim/ não/ não sei", onde uma das alternativas deve ser assinalada para cada item, como é mostrada abaixo.

\section{Alocação de tratamento:}

A. Foi realizado um método de aleatorização? Sim / Não / Não Sei

B. A alocação do tratamento foi sigilosa? Sim / Não / Não Sei

2. A entrada no estudo, os grupos eram similares com relação aos indicadores prognósticos mais importantes?

Sim / Não / Não Sei

3. Os critérios de elegibilidade foram especificados?

Sim / Não / Não Sei

4. Os avaliadores dos desfechos foram mascarados?

Sim / Não / Não Sei

5. Os que ofereceram a intervenção eram mascarados?

Sim / Não / Não Sei

6. Os pacientes eram mascarados em relação a intervenção?

Sim / Não / Não Sei

7. Em relação às medidas de desfecho primário, foram relatadas estimativas das medidas de tendência central e variabilidade?

Sim / Não / Não Sei

8. A análise foi realizada pela intenção de tratar? Sim / Não / Não Sei

\section{RESULTADOS}

Dos 207 trabalhos localizados na leteratura, por meio dos descritores utilizados, 50 foram excluídos devido suas referências apresentarem erros de confecção, por exemplo, ausência do número do fascículo, nome dos autores e outras falhas, impossibilitando a obtenção dos artigos na integra para análise. Os 157 artigos restantes foram analisados, sendo selecionados 22 artigos pois, apenas estes utilizaram o recurso "fisioterapia aquática" para tratamento, os demais utilizaram a "hidro-terapia" como meio de intervenção, ou seja, qualquer forma de aplicação externa de água, não condizente com o objetivo deste estudo. Dos 22 artigos, 
preencheram os critérios de inclusão do estudo 6 trabalhos, ou seja, eram ensaios clínicos aleatórios, os demais eram relatos de caso ou opinião do especialista. A catalogação e as características dos 6 artigos incluídos nesta revisão são apresentadas nos quadros 1 e 2 respectivamente.

\section{DISCUSSÃO}

Dos 6 (seis) artigos selecionados e analisados, nenhum dos artigos apresentou:

A. Sigilo na alocação do tratamento, que os desenhos de ensaios clínicos futuros sobre fisioterapia aquática devem descrever, ou seja, uma seqüência aleatória de tarefa gerada por uma pessoa independente do estudo (esta não pode ter informações sobre quais pacientes foram incluídos no grupo de estudo, e também não pode influenciar nas seqüências das tarefas realizadas pelos pacientes), a fim de melhorar a qualidade dos estudos sobre esta temática.

B. Mascaramento dos pacientes em relação à intervenção, isto se deve ao fato de que os pacientes submetidos a procedimentos fisioterapêuticos têm conhecimento do tratamento, exceto quando submetidos a tratamentos eletroterápicos sem contato.

Um estudo apresentou o método da aleatori- zação $^{[4]}$, mostrando que este não é um método utilizado regularmente em ensaios clínicos sobre este assunto devendo, estudos futuros utilizar-se deste método para a alocação dos pacientes.

Houve, também apenas um estudo onde os avaliadores dos desfechos eram mascarados ${ }^{[5]}$, sendo este item de grande importância, pois quando isto não ocorre, os resultados do estudo podem ser tendenciosos.

Em relação ao mascaramento sobre os terapeutas que ofereceram a intervenção, apenas um dos estudos ${ }^{[4]}$ utilizou-se deste critério, pois dificilmente os terapeutas pesquisadores não irão fazer parte da aplicabilidade do procedimento do estudo. Há, portanto, a necessidade da participação de terapeutas que não tenham conhecimento sobre os objetivos do estudo.

A maioria dos estudos apresentou grupos similares em relação aos indicadores prognósticos mais importantes $[4,5,7,8]$ dos pacientes e, todos os estudos ${ }^{[4-9]}$ apresentaram critérios de elegibilidade especificados, intenção de tratar e, foram relatadas estimativas das medidas de tendência central e variabilidade nas medidas de desfecho primário, propiciando, assim estudos de melhor qualidade.

Em relação ao trabalho de revisão sistemática, espera-se encontrar evidências científicas a respeito do assunto pesquisado. Para tanto, ob-

\begin{tabular}{|c|l|l|c|c|c|c|}
\hline \multirow{2}{*}{ ECA } & \multirow{2}{*}{ Ano } & \multirow{2}{*}{ Autores } & \multicolumn{2}{|c|}{ Doenças Neurológicas } & \multicolumn{2}{|c|}{ N } \\
\cline { 3 - 6 } & 1994 & $\begin{array}{l}\text { Prins JH, Hartung GH, Merritt } \\
\text { DJ, Blancq BS, Goebert DA. }\end{array}$ & poliomielite & poliomielite & 9 & 7 \\
\hline 1 & 1996 & $\begin{array}{l}\text { Dorval G, Tetreault S, } \\
\text { Caron C. }\end{array}$ & PC & PC & 10 & 10 \\
\hline 2 & 1998 & $\begin{array}{l}\text { Hutzler Y, Chacham A, } \\
\text { Bergman U, Szeinberg A. }\end{array}$ & PC & PC & 23 & 23 \\
\hline 4 & 1998 & $\begin{array}{l}\text { Hutzler Y, Chacham A, } \\
\text { Bergman U, Reches I. }\end{array}$ & PC & PC & 23 & 23 \\
\hline 5 & 1998 & Zamparo P, Pagliaro P. & EM, AVC, MP & EM, AVC, MP & 23 & 23 \\
\hline 6 & 1999 & Pagliaro P, Zamparo P. & $\begin{array}{c}\text { MP, EM, } \\
\text { AVC, TCE }\end{array}$ & $\begin{array}{c}\text { MP, EM, } \\
\text { AVC, TCE }\end{array}$ & 26 & 26 \\
\hline
\end{tabular}

$\mathrm{PC}=$ paralisia cerebral, $\mathrm{EM}=$ esclerose múltipla, $\mathrm{AVC}=$ acidente vascular cerebral, $\mathrm{MP}=$ mielopatia, $\mathrm{TCE}=$ traumatismo crânio-encefálico.

Quadro 1. Ensaios clínicos aleatórios (ECA) segundo ano de publicação, autores, doenças neurológicas dos grupos de estudo (GE) e controle (GC) e número de pacientes (N) pertencentes aos GE e GC. 


\begin{tabular}{|c|c|c|c|}
\hline ECA & Objetivos do Estudo & $\begin{array}{l}\text { Tipo de intervenção com } \\
\text { Fisioterapia Aquática }\end{array}$ & Conclusões do Estudo \\
\hline 1 & $\begin{array}{l}\text { Determinar o efeito } \\
\text { doprograma de exercícios } \\
\text { aquáticos sobre a força } \\
\text { muscular. }\end{array}$ & $\begin{array}{l}\text { Exercícios ativo-resistidos utilizando } \\
\text { equipamentos como "palmar" e } \\
\text { "nadadeira". }\end{array}$ & $\begin{array}{l}\text { O grupo de estudo } \\
\text { apresentou aumento da força } \\
\text { muscular quando comparado } \\
\text { ao grupo controle. }\end{array}$ \\
\hline 2 & $\begin{array}{l}\text { Comparar o impacto acurto e } \\
\text { longoprazo de dois programas } \\
\text { aquáticos específicos em } \\
\text { relação a autoestima e } \\
\text { independência funcional e, } \\
\text { averiguar a efetividade da } \\
\text { fisioterapia aquática. }\end{array}$ & $\begin{array}{l}\text { Programa 1. Exercícios de } \\
\text { aquecimento e relaxamento, jogos } \\
\text { aquáticos e natação adaptada sem } \\
\text { objetivos terapêuticos. } \\
\text { Programa 2. Exercícios de } \\
\text { Aquecimento, relaxamento, ativos } \\
\text { individuais e em grupo com objetivos } \\
\text { terapêuticos gerais e específicos. }\end{array}$ & $\begin{array}{l}\text { Houve aumento na pontuação } \\
\text { das escalas de auto-estima e } \\
\text { independência funcional entre } \\
\text { os grupos de estudo e } \\
\text { controle, mas não houve } \\
\text { diferença significativa entre } \\
\text { os grupos. }\end{array}$ \\
\hline 3 & $\begin{array}{l}\text { Averiguar o efeito do } \\
\text { programa sobre a função } \\
\text { respiratória e avaliar o } \\
\text { desempenho de habilidade de } \\
\text { orientação aquática. }\end{array}$ & $\begin{array}{l}\text { Exercícios individuais de habilidade } \\
\text { de orientação aquática, exercícios } \\
\text { em grupo e de coordenação motora. }\end{array}$ & $\begin{array}{l}\text { Foi sugerido que a aplicação } \\
\text { associada dos procedimentos } \\
\text { de solo e água agem } \\
\text { beneficamente sobre a função } \\
\text { respiratória dos pacientes. }\end{array}$ \\
\hline 4 & $\begin{array}{l}\text { Avaliar as alterações quanto } \\
\text { às funções motoras e quanto } \\
\text { ao autoconceito de crianças } \\
\text { submetidas a um programa de } \\
\text { tratamento. }\end{array}$ & $\begin{array}{l}\text { Atividades aquáticas individuais } \\
\text { consistindo de exercícios de } \\
\text { habilidade de orientação aquática. }\end{array}$ & $\begin{array}{l}\text { Não houve alteração significativa } \\
\text { na escala de autoconceito e } \\
\text { houve melhora em relação às } \\
\text { habilidades aquáticas em ambos } \\
\text { os grupos. }\end{array}$ \\
\hline 5 & $\begin{array}{l}\text { Analisar o gasto energético na } \\
\text { marcha subaquática de pacientes } \\
\text { hemiparéticos, paraparéticos e } \\
\text { com alterações neuromotoras por } \\
\text { esclerose múltipla após programa } \\
\text { de fisioterapia aquática. }\end{array}$ & $\begin{array}{l}\text { Marcha subaquática, } \\
\text { grupo A: velocidade pré-determinada e } \\
\text { grupo B: velocidade eleita pelo próprio } \\
\text { paciente. Nado adaptado. }\end{array}$ & $\begin{array}{l}\text { A fisioterapia aquática foi } \\
\text { recomendada aos pacientes, } \\
\text { uma vez que melhorou às } \\
\text { características biomecânicas } \\
\text { da marcha e diminuiu seu } \\
\text { gasto energético. }\end{array}$ \\
\hline 6 & $\begin{array}{l}\text { Avaliação quantitativa do reflexo } \\
\text { miotático dos músculos do } \\
\text { quadríceps após programa de } \\
\text { fisioterapia aquática. }\end{array}$ & $\begin{array}{l}\text { Exercícios passivos, ativos, de } \\
\text { coordenação motora, marcha } \\
\text { subaquática e nado adaptado } \\
\text { quando possível. }\end{array}$ & $\begin{array}{l}\text { Não houve evidência da } \\
\text { Efetividade da fisioterapia } \\
\text { aquática sobre os pacientes } \\
\text { estudados. }\end{array}$ \\
\hline
\end{tabular}

Quadro 2. Relação de ensaios clínicos randomizados segundo os objetivos, tipo de intervenção e conclusões dos estudos.

jetiva-se agrupar dados suficientes em número de amostras, com homogeneidade entre elas e rigor científico dos protocolos utilizados para submetêlos a uma avaliação meta-analítica. Acredita-se que somente assim haverá evidência de que o tratamento pesquisado tenha efetividade. Neste estudo não foi possível submeter os ECA selecionados a uma avaliação meta-analítica, devido estes utilizarem métodos diferentes, impossibilitando alocá-los em um único grupo para análise.

No entanto, justifica-se a continuidade da prática da fisioterapia aquática, pois, os estudos já realizados não apontaram malefícios desta intervenção nos pacientes com doença neurológica.

\section{CONCLUSÕES}

1. Concluímos que as evidências na literatura são fracas quanto aos benefícios da fisioterapia aquática quando aplicada aos pacientes com doença neurológica e comprometimento motor.

A elaboração de ensaios clínicos aleatórios para avaliar o efeito da fisioterapia aquática em pacientes com doença neurológica específica é fundamental na decisão da prática fisioterapêutica. 


\section{REFERÊNCIAS BIBLIOGRÁFICAS}

1. Becker BE, Cole AJ. Terapia Aquática Moderna. São Paulo: Manole; 2000. p.20-1.

2. Campion MR. Hidroterapia: Princípios e Prática. São Paulo: Manole; 2000. p. 185-6.

3. Verhagen AP, De Vet HCW, De Bie RA, Kessels AGH, Boers M, Bouter LM, Knipschild PG. The Delphi list for quality assesment of randomized clinical trials for conducting sistematic reviews developed by Delphi consensus. J Clin Epidemiol 1998;51:1235-41.

4. Hutzler Y, Chacham A, Bergman U, Szeinberg A. Effects of a movement and swimming program on vital capacity and water orientation skills of a children with Cerebral Palsy. Dev Med Child Neurol 1998;40:176-81.

5. Hutzler Y, Chacham A, Bergman U, Reches I. Effects of a movement and swimming program on the water orientati- on skills and self - concept of kindergarten children with Cerebral Palsy. Percep Mot Skills 1998;86:111-8.

6. Dorval G, Tetreault S, Caron C. Impact of aquatic programmes on adolescents with cerebral palsy. Occup Ther Int 1996;3: 241-61.

7. Zamparo P, Pagliaro P. The energy cost of level walking before and after hydrokinesy therapy in patients with spastic paresis. Scand J Med Sci Sports 1998; 8:222-8.

8. Prins JH, Hartung GH, Merrit DJ, Blancq RJ, Goebert DA. Effect of aquatic exercise training in person with poliomyelitis disability. Sports Med 1994;5:29-39.

9. Pagliaro P, Zamparo P. Quantitative evaluation of the stretch reflex before and after hydrokinesy therapy in patients affected by spastic paresis. J Electromyogr Kinesiol 1999;9:141-8. 